\title{
Development of an edge sensor based on polyview optics and laser triangulation principle
}

\author{
Yinan Li, Hagen Bossemeyer, Markus Kästner, Eduard Reithmeier ${ }^{a}$ \\ ${ }^{a}$ Institute of Measurement and Automatic Control, Gottfried Wilhelm Leibniz Universität \\ Hannover, Nienburger Str. 17, 30167 Hannover
}

\begin{abstract}
Common 2D laser line triangulation sensors allow a 2D profile measurement in a single line. To scan samples with great curved surfaces like edges, a single laser line triangulation sensor is insufficient. To measure the entire form of such an edge, it normally requires either multiple measurements of one single sensor or a multi sensor system. For this reason, we developed an edge measurement sensor based on an in-house designed polyview optics and the well-known laser triangulation principle. The new developed edge measurement sensor is capable of measuring the object over a $180^{\circ}$ field of view (FOV). The configuration, the calibration process and the measurement results of this edge sensor will be discussed in this paper.
\end{abstract}

Keywords: 3D measurement, optical systems for inspection and measurements, system calibration

\section{INTRODUCTION}

Laser line triangulation sensors as non-contact measurement devices are very desirable in many inspection and measurement situations. Compared to common sensors like capacitive, inductive or ultrasonic devices, laser line triangulation sensors are available with longer working distances, higher resolutions and accuracy specifications. In different applications and diverse industry sectors, the sensors are available with a wide variety of configurations and specifications which replace and outperform older types of contact measurement sensors. However, the common laser line triangulation sensors have a geometrical optics limit for the measurement range and direction. The measurement range of a single sensor is limited by the rotation angle between the imaging detector and the projected structured laser light. The measurement direction is limited by the $180^{\circ}$ field of view (FOV) of the imaging lens and the 2D detector, which has flat light sensitive elements like CCDs or CMOS. In order to scan samples with great curved surfaces like edges, a single laser line triangulation sensor is not capable of measuring the entire edge form of the object in a single scanning. In this case, an entire 3D edge measurement requires either multiple measurements of one single sensor from different directions or a multi-sensor system. ${ }^{12}$ Fig. 1 shows a typical combination of dual laser profile sensors for edge measurement.

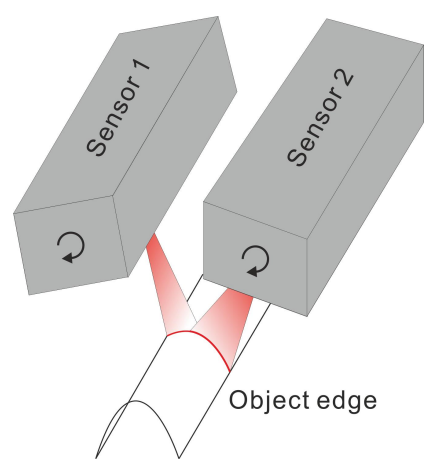

Figure 1. Edge sensor using the combination of two laserline sensors

Send correspondence to Yinan.li@imr.uni-hannover.de; phone +49511 7623236

Optical Metrology and Inspection for Industrial Applications IV, edited by Sen Han, Toru Yoshizawa, Song Zhang,

Proc. of SPIE Vol. 10023, 100231E · @ 2016 SPIE · CCC code: 0277-786X/16/\$18 · doi: 10.1117/12.2247007 
The purpose of this paper is to explain the development of an edge sensor based on an in-house designed polyview optical lens and the well-known laser triangulation principle. Utilizing a polyview lens system, the FOV of a single camera can be divided into two sub FOVs, which are allocated to different measurement directions. Using this advantage, the measurement system allows a measurement FOV over $180^{\circ}$.

\section{EXPERIMENTAL SETUP}

The edge measurement system shown in Fig. 2 is set up with two red laser line diodes (laser class 1) (1), a polyview lens system (2 3), a camera lens (4) and an industrial CCD camera (5). To project an over $180^{\circ}$ laserline on the target object, the two laser diodes are positioned using the ball and socket mount in two different directions in front of the target object. The thickness of the projected laserlines within the focus range of $10 \mathrm{~mm}$ to $15 \mathrm{~mm}$ is less than $0.15 \mathrm{~mm}$ and has a $90^{\circ}$ angle of radiation. Moreover, the projected laser lines are adjusted to overlap in one resulting line with the same horizontal angle. The overlapping laser lines are also angled vertically to the optical axis of the camera, due to the principle of laser triangulation. The adjustment of the laser lines should be carried out carefully, as diverging or crossed laser lines can lead to major measurement errors.

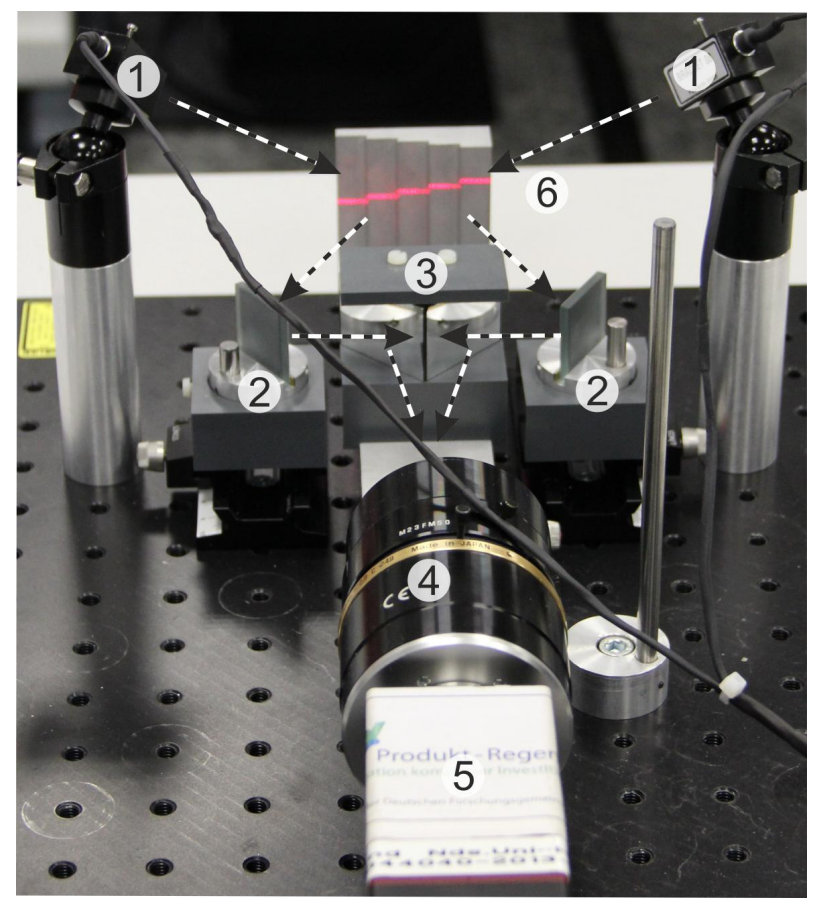

Figure 2. Experimental setup of the edge measurement system: (1) red line diode laser, (2) lateral mirror set of the polyview lens, (3) central mirror set of the polyview lens, (4) camera lens, (5) industrial CCD camera, (6) target object

The projected laser lines on the target object are reflected as an incident light with an over $180^{\circ} \mathrm{FOV}$, which is deflected passing the in-house designed polyview optical lens and a $50 \mathrm{~mm}$ camera lens, and is finally focused on the CCD Sensor of the camera. The designed polyview optical lens consists of a lateral mirror set (2) in Fig. 2 , where two mirrors are positioned parallel to the image plane and are angled at approximately $225^{\circ}$ to the optical axis as a viewing angle, and a central mirror set (3) in Fig. 2, where two further mirrors are positioned at the central position of the optical axis of the image plane and are levelled $45^{\circ}$ horizontally to the optical axis. The whole polyview mirror sets are placed for a short working distance between the mirror sets and the target object, in order to decrease the overlapping range of the separate images. The incident light passing the polyview lens is separated by the viewing angle of the lateral mirror set firstly, and is combined afterwards by the central mirror set in the same direction (see the arrows in Fig. 2). 
The deflected light passing the polyview lens is focused on the camera using a camera lens, which can be adjusted to different working distances and magnifications. The closest focusing distance of $150 \mathrm{~mm}$ at the given focal length allows the acquisition of small details on edge geometries with a larger magnification. However, the larger magnification can countervail the amount of depth of field (DOF) and the limited DOF determines the vertical measurement range. At this situation, the focus distance of this camera lens is adjusted up to $250 \mathrm{~mm}$ and its aperture is adjusted by f11 as a compromise between DOF and light gathering, so that the focus ranges of the camera and the laserline diodes can be adjusted in the same range to allow a large measurement range and a high resolution. The mirrors and camera are mounted on a optical rail system, so that the optical axis of the camera can be adjusted precisely vertically to the mirror sets.

Finally, the reflected laserline passing the polyview lens and camera lens is captured by a 5MP monochrome industrial camera, which is connected to a PC using the FireWire interface (800Mbits/sec). The captured image of the real camera (the industrial camera) includes two sub-images obtained by the two different viewing angles of the target object (see the original image in Fig. 3). In this situation, the real camera can be considered as two separated virtual cameras, which can capture the images from two individual viewing angles. Utilizing the captured image of the laserline and the triangulation principle, the geometric information of the virtual cameras can be calculated separately. After the calibration of the measurement system, the whole measurement of an edge can be registered from the two separate measurement results.

\section{CALIBRATION OF THE MEASUREMENT SYSTEM}

To calibrate the virtual cameras, a set of images of a black and white checkerboard pattern described in the literature $^{3}$ are captured as a calibration data set. The captured image of the real camera includes two sub-images of the virtual cameras. Before the camera calibration, the original image should be segmented into two individual images. The middle of the original image has few available information, because the central mirror set has a seam zone, where the reflected light is none or high distorted. For this reason, two boxes are defined as the segmented range of the sub-images (see Fig. 3) which are smaller than half of the original image. Using this segmentation method, the calibration images are divided into a data set of the left sub-images and a data set of the right sub-images.

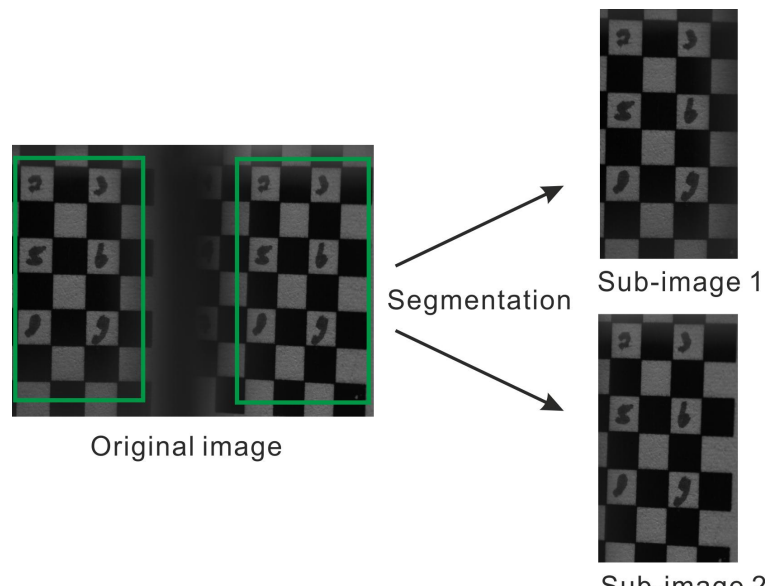

Figure 3. Segmentation of the original image of a checkerboard pattern

\subsection{CALIBRATION OF THE VIRTUAL CAMERA SYSTEM}

Camera calibration is a necessary step to ensure a high measurement accuracy of the laser triangulation system. Using the segmented checkerboard images, the individual virtual camera systems can be calibrated by the method of Zhang, ${ }^{45}$ which evaluates camera parameters and lens distortion coefficients using the captured images of the same checkerboard pattern at multiple orientations. The cameras are modeled with the help of the pinhole camera model, which describes a 2D image plane transformation from a 3D space with an influence of lens 
distortions (e.g. the typical barrel distortion, pincushion distortion and mustache distortion). The used lens distortion model is presented in the mathematical description in the literature. ${ }^{6}$ After the camera calibration, the intrinsic parameters matrix $\boldsymbol{K}$ and the extrinsic parameters matrix $\boldsymbol{P}$ can be estimated as follows:

$$
\boldsymbol{K}=\left[\begin{array}{ccc}
f_{x} & s & c_{x} \\
0 & f_{y} & c_{y} \\
0 & 0 & 1
\end{array}\right], \boldsymbol{P}=\boldsymbol{K}(\boldsymbol{I} \mid \mathbf{0})\left[\begin{array}{cc}
\boldsymbol{R} & \boldsymbol{t} \\
\mathbf{0} & \mathbf{1}
\end{array}\right]=\boldsymbol{K}(\boldsymbol{R} \mid \boldsymbol{t}),
$$

where $f_{x}, f_{y}$ are the focal length in pixels of the camera in the $\mathrm{x}$ and y direction, $c_{x}, c_{y}$ are the optical centers transformed in pixels coordinates and $s$ is the angle coefficient between the $\mathrm{x}$ and y sensor axes; in addition, $\boldsymbol{R}$ is the rotation matrix for the extrinsic parameters and $\boldsymbol{t}$ is the translation vector. Moreover, the radial and tangential distortion coefficients of the lens can be represented as follows:

$$
\left\{\begin{array}{l}
x_{d}=x_{u}\left(1+k_{1} r^{2}+k_{2} r^{4}+k_{3} r^{6}\right)+2 p_{1} x y+p_{2}\left(r^{2}+2 x^{2}\right) \\
y_{d}=y_{u}\left(1+k_{1} r^{2}+k_{2} r^{4}+k_{3} r^{6}\right)+p_{1}\left(r^{2}+2 y^{2}\right)+2 p_{2} x y
\end{array}\right.
$$

where $k_{1}, k_{2}, k_{3}$ are the coefficients of the radial distortion and $p_{1}, p_{2}$ are the coefficients of the tangential distortion. These lens distortion coefficients can be estimated by the camera calibration in the camera model. Using these coefficients, the captured original image can be undistorted for the triangulation calculation. Moreover, the relative distance between the camera and calibration body is further calibrated using a step standard, which consists of steps with $3 \mathrm{~mm}$ height and $5 \mathrm{~mm}$ length.

\subsection{REGISTRATION OF THE MEASUREMENT DATA}

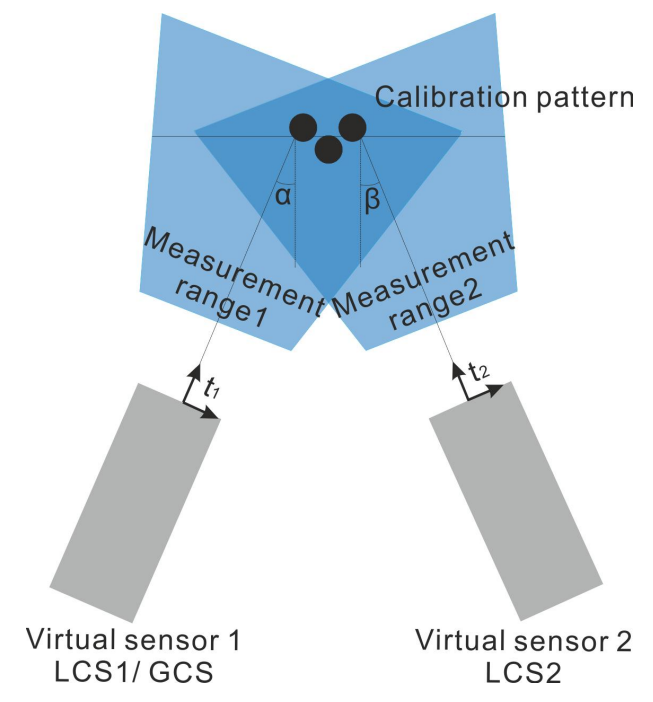

Figure 4. Calibration of the coordinates of the virtual sensors

The two virtual cameras and laser diodes can be seen as two separated laser triangulation sensors, where each sensor builds an individual local sensor coordinate system (LCS). Fig 4 shows the coordinates and the measurement ranges of the sensors. To register both measurement data from LCSs into a single global sensor coordinate system (GCS), we calibrate the sensors using a calibration pattern which consists of three cylinders. From the measurement data of the virtual sensors, the rotation angles $\alpha, \beta$ and the translation vectors $\boldsymbol{t}_{\mathbf{1}}, \boldsymbol{t}_{\mathbf{2}}$ between the both sensors can be determined. The coodinates of one of the virtual sensors is defined as the coordinate origin of the system. In this system, the coordinate of sensor 1 is defined as the coordinate origin shown in Fig. 4. Utilizing the known rotation and translation parameters, the GSC can be converted by the Euclidean transformation 


$$
T_{E}=\left[\begin{array}{ll}
R & \boldsymbol{t}
\end{array}\right]=\left[\begin{array}{cc}
\cos \Theta & -\sin \Theta \\
\sin \Theta & \cos \Theta
\end{array}\right]+\left[\begin{array}{c}
t_{x} \\
t_{y}
\end{array}\right],
$$

where the relative rotation angle is equal to $\Theta_{21}=\alpha+\beta$ and the relative translation is equal to $\boldsymbol{t}_{\mathbf{2 1}}=\boldsymbol{t}_{\mathbf{2}}-\boldsymbol{t}_{\mathbf{1}}$. Due to the static measurement, the calibration process is available only for the $2 \mathrm{D}$ cross section range. The calibration in the vertical direction is not considered in this situation.

\section{RESULTS}

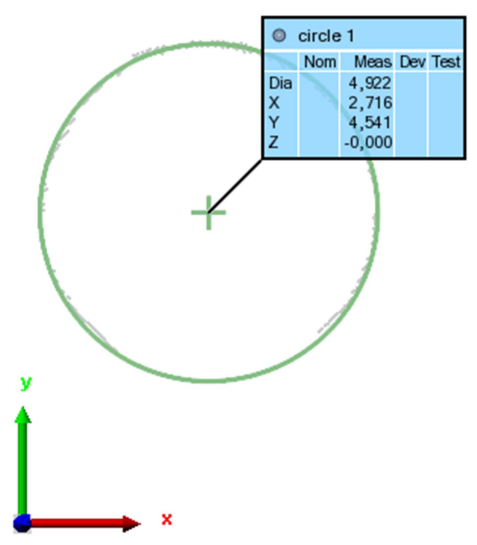

Figure 5. Measurement of a cylinder standard

The edge measurement system described in Sec. 2 using the diode lasers, the polyview lens and the camera system allows a measurement range FOV of over $180^{\circ}$. This sensor system is suited for the scanning and inspection of edge geometries. To verify the accuracy of the measurement for an edge object, a cylinder standard with a $5 \mathrm{~mm}$ diameter is measured. The measured cross section shows not a whole circle form in Fig. 5 , where the part of the object behind the projected laser line cannot be scanned. To calculate the measurement error, the measurement data is fitted using a circle fitting, where the fitting circle diameter is compared with the known cylinder diameter. Statistical analysis of the accuracy of the measurement shows that, the measurement system with a $0.06 \mathrm{~mm}$ resolution is capable of determining the cylinder diameter, while the measured diameter has a low error less than $0.01 \mathrm{~mm}$.

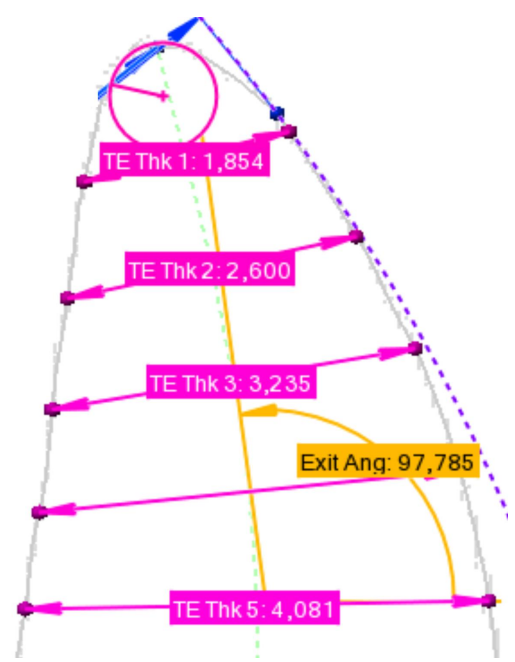

Figure 6. Measurement of an edge of a turbine blade 
In addition, to evaluate the performance of the system, we used it to scan the trailing edge of a worn turbine blade. Fig. 6 shows the measured range of the edge, which covers more than $180^{\circ}$ on the edge. The geometric characteristics of the edge can be determinated using an airfoil analysis tool, where the typical characteristics like the trailing edge radius, the exit angle and the thickness of the edge are determined and shown in Fig. 6. These extracted values can be further used as input data for aerodynamic CFD (Computational Fluid Dynamics) simulations.

\section{CONCLUSION AND OUTLOOK}

In this article, we propose an edge measurement system with an over $180^{\circ} \mathrm{FOV}$, which is designed based on polyview optics and laser triangulation principle. It is capable of measuring an edge geometry in a single scanning. The construction of the experiment setup, the calibration of the camera and the registration of the measurement data are discussed in depth in Sec. 3. The experimental results in Sec. 4 show a comparison measurement with a cylinder standard and an edge measurement of a turbine blade.

The actual measurement system is only available for a static cross section measurement. In order to further scan an edge in a larger range or more dimensions, the edge sensor can be installed on a linear motor stage to scan in the vertical direction or installed on a rotation stage for ring scanning. Moreover, the sensor can be installed on an industrial robot based guided system for an automatic measurement. Moreover, this edge sensor can also be integrated in our multi-sensor-system consisting of a fringe projection system ${ }^{7}$ and a compact low coherence interferometer. ${ }^{8}$

\section{ACKNOWLEDGMENTS}

We gratefully acknowledge that this project "Multiscale Measurement of Blade Geometries with Robot-Supported, Laser-Positioned Multi-Sensor-Techniques" has been funded by the German Research Foundation (DFG) within the Collaborative Research Center 871 Regeneration of Complex Capital Goods.

\section{REFERENCES}

[1] Zhang, W., Zhao, H., and Zhou, X., "Multiresolution three-dimensional measurement system with multiple cameras and light sectioning method," Optical Engineering 49(12), 123601-123601 (2010).

[2] Y. Li, M. Kästner, E. R., "Edge measurement based on multi laser triangulation sensors," 117. Jahrestagung der DGaO (Deutsche Gesellschaft für angewandte Optik) 21, B5 (2016).

[3] Hartley, R. and Zisserman, A., [Multiple view geometry in computer vision], Cambridge university press (2003).

[4] Zhang, Z., "A flexible new technique for camera calibration," IEEE Transactions on pattern analysis and machine intelligence 22(11), 1330-1334 (2000).

[5] Zhang, Z., "Flexible camera calibration by viewing a plane from unknown orientations," in [Computer Vision, 1999. The Proceedings of the Seventh IEEE International Conference on], 1, 666-673, Ieee (1999).

[6] Fryer, J. G. and Brown, D. C., "Lens distortion for close-range photogrammetry," Photogrammetric engineering and remote sensing $\mathbf{5 2}(1), 51-58$ (1986).

[7] Schlobohm, J., Li, Y., Pösch, A., Langmann, B., Kästner, M., and Reithmeier, E., "Multiscale optical inspection systems for the regeneration of complex capital goods," Procedia CIRP 22, 243-248 (2014).

[8] Li, Y., Kästner, M., and Reithmeier, E., "Development of a compact low coherence interferometer based on gpgpu for fast microscopic surface measurement on turbine blades," in [SPIE Optical Metrology], 95250R95250R, International Society for Optics and Photonics (2015). 